\title{
When and Where in Skill Memory Consolidation: Neuro-Behavioral Constraints on the Acquisition and Generation of Procedural Knowledge.
}

\author{
Avi Karni* Maria Korman ${ }^{\dagger}$ \\ (*)Department of Human Biology \& the E.J. Safra Brain Research Center, University of Haifa, \\ Israel; $(\dagger)$ Technion, Haifa, Israel \\ E-mail: Avi.karni@yahoo.com,korman.maria@gmail.com
}

\begin{abstract}
Compelling behavioral and neuro-imaging data suggest that the retention and perfection of skills (procedural, "how to" knowledge) reflects long-lasting experience-driven changes in the brain's organization (neural plasticity). Two corollaries require consideration in designing effective skill learning programs. i) Neuro-behavioral constraints, imposed on whether neuronal plasticity is triggered and allowed to proceed, must be satisfied; otherwise, the skill may fail to consolidate into long-term memory. These include the amount of task iterations afforded, task scheduling, behavioral relevancy and the degree of consistency of the to-be-learned experience over a required timewindow. ii) The performance of a given task reflects qualitatively different task solution routines in different phases of experience. Practice, given time and sometimes time-in-sleep, can trigger processes whereby new procedural knowledge and qualitative changes in task solution, emerge and consolidate. These emerging changes in procedural knowledge result in differences in the ability to transfer gains, across stimulus, context and task parameters.
\end{abstract}

Our aim here is to present a very brief account of our current view of procedural learning and procedural memory consolidation as emerging from a number of studies addressing the characteristics of human skill learning. We present a number of points which we believe are of relevance to the understanding of the biological mechanisms and specifically, the neurobehavioral constraints imposed by these mechanisms on skill learning and skill memory. These constraints require consideration if we are to improve and perhaps even optimize skill teaching protocols and skill learning programs. The references provided, mainly from our own work on perceptual learning and motor skill acquisition can be consulted for perusing the actual data and as pointers to many related studies and papers that inspired us.
A widely accepted view is that skills and habits procedural, "how to" knowledge - are organized in memory in a manner which is quite distinct from that of declarative, "what" knowledge (knowledge of facts and singular events) and are retained in an outstandingly robust manner (Squire, 1986; Dudai, 2004). It is clear, nevertheless, that the two memory systems interact in healthy, typical adults (Albouy et al., 2008). A somewhat different perspective on the two long-term memory systems is that while the declarative memory system is geared to retain sparsely occurring and even singular experiences, the declarative memory system is triggered if and only when a given experience is repeated; preferably in a consistent manner. Thus, declarative memory can be viewed as a memory system evolved from the need to retain singular events (e.g., painful event that one would not want repeated) and arbitrary associations, while procedural memory reflects the evolving 'deep' knowledge of the structure of the relevant environment and context, as well as the statistics of repeated experiences. In the context of skill learning, a training experience will often result in some trace of declarative memory, but if training is continued, i.e., more iterative experience on the task is afforded and experience accumulates, the neural mechanisms (presumably Hebbian in character (Viana \& Prisco, 1984) subserving the generation of long-term procedural memory can be triggered.

An important advancement in our understanding of the biology of procedural memory comes from animal and human studies that clearly indicated that the establishment of long-term procedural memory is subserved by functional and structural changes within the brain systems involved in the performance of the task, i.e., activated by the repeated experience (Karni, 1996; Merzenich, 1998). Moreover, there is evidence suggesting that the neural changes triggered by repeated experience affect two distinct levels of brain representation. Repeated experience can result: i. in neuronal changes at local processing levels that are

This is an Open Access article distributed under the terms of the Creative Commons Attribution-Noncommercial License 3.0, which permits unrestricted use, distribution, and reproduction in any noncommercial medium, provided the original work is properly cited. 
critically involved in task performance at the given phase of experience (synaptic consolidation) (Dudai, 2004), and ii. in a shift or re-setting in the task and stimulus relevant processing streams (systems consolidation) (Korman, 2005).

Synaptic and systems level changes can be expressed in human behavior as qualitative changes in performance. The changes at the system level, however, indicate that a different task solution is implemented. To illustrate the point, we consider the case of handwriting. We tend to assume that handwriting is ' $a$ ' task, complex but nevertheless a coherent skill (or a 'type' of experience). We propose, however, that because at different levels of experience the experience engages different brain representations (systems level plasticity) the actual skill (procedural knowledge) changes. Thus, we argue, handwriting for a high frequency word may be very different from handwriting of a low frequency word, even for an identical target letter embedded in both words (Balas et al, 2007). We further propose that these experiencedriven shifts in the brain representation of the task are reflected in actual performance, as we and other have shown. First, it is well established that the performance at a novice level is qualitatively different from performance after extensive practice; one can show however, that new, specific, task solution routines and novel movement elements are generated and implemented in the latter stage (Sosnik et al, 2003; Korman et al, 2003; Rozanov 2010). Second, the system level changes are also reflected in changes in the ability to transfer gains, across shifting stimulus, context and task parameters (skill specificity profile), in different phases of experience (Karni \& Sagi, 1993; Karni, 1996; Korman et al, 2003). We propose that the ability to transfer the gains accrued in training can change because different representations, even within a given processing stream, are tuned to different aspects of the experience (Karni, 1996).

If one accepts that the generation of skill necessitates neural changes, several critical questions present themselves. Firstly, where in the brain do experiencedriven changes occur? As we have argued above, this is not a straightforward question to answer because we think that the answer depends on when (or how much practice experience was afforded) and on what (the nature of the task and the particulars of the training experience) because procedural knowledge is dynamic and can evolve towards a task specific solution. Emerging from our and others' work in recent years is the notion that experience-driven changes can occur even at low-level cortical processing stages but within the processing stream involved in the execution of the to be learned task (For example, Karni et al, 1995,
1998; Schwartz et al, 2005; Blake et al, 2005; and see also M. Greenlee's paper in the current proceedings). Based on a principle of parsimony, it has been proposed (Karni, 1996), that experience dependent changes would occur at the lowest possible level in which task parameters which are critical for task performance are differentially represented by a group of neurons or a local circuit (the Critical Level theory). The critical level for task representation reflects not only task parameters and the nature of the training experience, but also of the amount of experience gained, because representations change. Thus, the level to be affected by plasticity is determined, according to the Critical Level hypothesis, by multiple factors such as the nature and structure of the training experience, the ability to represent the relevant aspects that a re critical to the task solution implemented and the task demands but also relates to the learners experiential "history" (e.g., prior experience with the target and related - in a neural representational sense - tasks) and needs (relevance, priority) (Karni, 1996). It is important to note that in line with the Critical Level theory and contrary to a widely held view, the more one practices, the less amenable are the skills to be expressed in novel (untrained) conditions: the more practice the more lower-level brain representations become engaged (e.g., Korman et al, 2003; Sosnik et al, 2003; Hauptmann \& Karni, 2002).

The second question called to mind is when do these changes occur? The studies addressing this question lead to the discovery of a latent memory consolidation phase in procedural learning (e.g., Karni \& Sagi, 1993; Karni, 1996, 1998; Korman et al., 2003, 2007; AdiJapha et al., 2009; Shadmehr R \& Brashers-Krug, 1997). During a few hours after the termination of the training experience memory processes, which were triggered in training, result in measurable changes in the brain as well as in task performance. The successful completion of procedural memory consolidation was shown, by us and others, to be in many cases sleep dependent (Stickgold et al, 2000; Walker et al., 2002; Korman et al., 2007). Nevertheless, sleep may be necessary only for memory consolidation in the motor domain (Korman et al, 2003, 2007; Walker, 2003). Several of our studies showed that the triggering and expression of consolidation phase gains depend on specific parameters of the training experience in the context of subsequent experiences occurring before time and sleep is afforded (e.g., amount of task iterations (Hauptmann \& Karni, 2002; Korman et al, 2003; Censor et al., 2007), sleep or time interval (Korman et al., 2003, 2007; Doyon, Korman et al., 2009), interference and scheduling (Korman et al., 2007, 
Balas et al., 2007); We would argue that these constitute likely parameters for optimizing skill learning protocols.

The notion that procedural skill learning is a multistaged, dynamic, process is based on extensive behavioral, functional imaging, electrophysiological, and cellular/molecular evidence (for some reviews see Willingham, 1998; Karni et al., 1998, Shadmehr \& Brashers-Krug, 1997; Hikosaka et al., 1999). Much of our work in recent years has been concerned with the characterization of a critical, time and time-in-sleep dependent, phase in the establishment of skill - the procedural memory consolidation phase (e.g., Karni \& Sagi, 1993, Karni et al, 1994, Korman 1998, 2007; Doyon et al, 2009). Nevertheless, we think, that the dynamic aspect of the process have not been taken into account in the cognitive skill learning literature. Specifically, the impact of the notion that the switch from one phase to the subsequent one is not linear or a simple function of the number of iterations as the "Power Law" model of skill learning suggests, is still to be felt. For example, it is clear that multi-session training does not constitute the sum of incremental gains of a number of single sessions of training on a task. Multi-session training can result in (numerous) qualitative shifts in the knowledge gained from the experience (Korman et al, 2003; Hikosaka et al., 1999).

A further important notion to have emerged in recent research is that skill learning, specifically, the generation of long-term procedural memory is under multiple control mechanisms (i.e., whether learning will occur is "gated") which are in effect, in varying modes, throughout the individuals' life time. It has been proposed (Karni, 1996; Keuroghlian, \& Knudsen, 2007) that some constraints on skill acquisition are required, in an ontogenetic sense, because skill and other types of habit learning represent a set of processes in which behaviorally important representations of experience, sometimes including low-level sensory and motor processing, are modified. Although most theories of skill acquisition assume that all skills are more alike than different to each other in terms of the characteristics of the skill acquisition process and the cognitive and brain processes subserving them, the evidence in support of this notion has been slow in developing. A review of the literature up to the $21^{\text {st }}$ century (Rosenbaum et al., 2001) suggests that intellectual (abstract, e.g., language, mathematics, logic) and perceptual and motor (simple "lower-level" but also complex artistic and professional, e.g., surgical procedures, sports and games) skills are acquired in fundamentally similar ways. One conceptual framework in which this apparent unity can be explained and some ground for empirical testing are afforded, is the notion that a limited repertoire of neuronal mechanisms of plasticity is at work in the generation of procedural (how to) knowledge (e.g., Karni, 1996). Thus, what is shared across different task domains is the process of skill acquisition, specifically, many aspects of the time-course of skill acquisition, and a number of general constraints on plasticity which need to be satisfied by the structure of the training experience, rather than the nature of the knowledge gained in different domains.

In sum, skill learning should be viewed as a dynamic, multi-phased and highly constrained process. Skills, even those engaged in what would seem to be, in cognitive analysis, very similar tasks and very similar task conditions, may be subserved by different (partially or completely non-overlapping) neuronal substrates at different levels experience. These changes involve different levels of structural resolution: from different synapses within a shared neuron to different columns in a shared brain area, to a difference in the network of brain areas engaged in the performance of the task. This 'tampering' with basic neuronal architecture is under strict constraints that must be satisfied before long-lasting changes are allowed. One trigger for these representation changes is the continuing demand for task performance and its perfection, over multiple time intervals.

We argue that it is important, to try and understand the behavioral and neural constraints on the acquisition of procedural knowledge and specifically the constraints on the triggering and the establishment of long-term procedural memory (i.e., procedural memory consolidation). The main motivation is the idea that brain plasticity can be better harnessed for improving skill acquisition across our life spans as well as for the rehabilitation of skills in individuals with developmental or acquired neurological deficits.

\section{References}

[1] L.R. Squire, Mechanisms of memory. Science, 1986, 232: 1612-1619.

[2] Y. Dudai The neurobiology of consolidations, or, how stable is the engram? Annu Rev Psychol. 2004;55:51-86.

[3] G. Albouy, V. Sterpenich, E. Balteau, G.Vandewalle, M. Desseilles, T. Dang-Vu, A. Darsaud, P. Ruby, PH Luppi, C. Degueldre, P. Peigneux, A. Luxen, P. Maquet. Both the hippocampus and striatum are involved in consolidation of motor sequence memory. Neuron. 2008 Apr 24;58(2):261-72.

[4] D. Viana, G. Prisco. Hebb synaptic plasticity. Prog Neurobiol. 1984; 22(2):89-102. 
[5] A. Karni The acquisition of perceptual and motor skills: A memory system in the adult human cortex. Cognitive Brain Research (1996):5(1), 39-48

[6] M. Merzenich Long-term change of mind. Science. 1998 Nov 6;282(5391):1062-3

[7] M. Korman, T. Flash \& A. Karni Synaptic \& system consolidation may explain the complex relationship between resistance to interference and the emergence of delayed gains in newly-acquired procedural memory (commentary on Walker MA: A refined model of sleep and the time course of memory consolidation). Behavioral and Brain Sciences. 2005 28:74-5.

[8] M. Korman, N. Raz, T. Flash and A. Karni Multiple shifts in the representation of a motor sequence during the acquisition of skilled performance. Proceedings of the National Academy of Science USA. 2003. 100(21):14292-14297.

[9] M. Balas, N. Roitenberg, N, Giladi \& A. Karni. (2007): Interference to consolidation phase gains in learning a novel movement sequence by handwriting: dependence on laterality and the level of experience with the written sequence. Experimental Brain Research. 2007 180(2):237-46.

[10] S. Rozanov, O. Keren, \& A. Karni The specificity of memory for a highly trained finger movement sequence: Change the ending, change all. Brain Research. 2010 May 17;1331:80-7.

[11] R. Sosnik, B. Hauptmann, A. Karni \& T. Flash. When a longer path is faster: The evolution of a movement primitive in the adult motor system. Experimental Brain Research. 2004 156:422-438.

[12] A. Karni and D. Sagi. The time course of learning a visual skill. Nature. 1993 365, 250-252.

[13] A. Karni, G. Meyer, P. Jezzard, M. M. Adams, R. Turner and L. G. Ungerleider. Functional MRI evidence for adult motor cortex plasticity during motor skill learning. Nature (1995): 377, 155-158.

[14] A. Karni, G. Meyer, C. Rey-Hippolito, P. Jezzard, M. M. Adams, R. Turner and L. G. Ungerleider The acquisition of skilled motor performance: fast and slow experience-driven changes in primary motor cortex. Proceedings of the National Academy of Science USA. 1998 95(3) 861-868.

[15] S. Schwartz, P. Maquet, C. Frith Neural correlates of perceptual learning: a functional MRI study of visual texture discrimination. Proc Natl Acad Sci U S A. 2002 Dec 24;99(26):17137-42. Epub 2002 Nov 21.

[16] DT Blake, F. Strata, R. Kempter, MM. Merzenich Experience-dependent plasticity in S1 caused by noncoincident inputs. $J$ Neurophysiol. 2005 Sep;94(3):2239-50.

[17] B. Hauptmann and A. Karni Primed to learn: saturation of repetition priming and the induction of long-term memory. Brain Research - Cognitive Brain Research 2002 13(3): 313-322.

[18] R. Stickgold, L. James, JA. Hobson Visual discrimination learning requires sleep after training. Nat.Neurosci. 2000; 3: 1237-8.

[19] MP. Walker, T. Brakefield, A. Morgan, JA. Hobson, R. Stickgold Practice with sleep makes perfect: sleepdependent motor skill learning. Neuron 2002; 35: 20511 .

[20] M. Korman, J. Doyon, J. Doljansky, J. Carrier, Y. Dagan and A. Karni. Daytime sleep condenses the timecourse of motor memory consolidation. Nature Neuroscience 2007 10(9):1206-13.

[21] R. Shadmehr, T. Brashers-Krug Functional stages in the formation of human long-term motor memory. Journal of Neuroscience 1997; 17: 409-19.

[22] E. Adi-Japha, A. Karni, A. Parnes, I. Loewenschuss, E. Vakil. A shift in task routines during the learning of a motor skill: group-averaged data may mask critical phases in the individuals' acquisition of skilled performance. Journal of Experimental Psychology: Learning, Memory \& Cognition 2008 Nov;34(6):154451.

[23] MP. Walker, T. Brakefield, JA Hobson, R. Stickgold. Dissociable stages of human memory consolidation and reconsolidation. Nature. 2003 Oct 9;425(6958):616-20.

[24] N. Censor, A. Karni, \& D. Sagi (2006): A link between perceptual learning, adaptation and sleep. Vision Research. 2006 46(23):4071-4.

[25] J. Doyon, M. Korman, A. Morin, V. Dostie, A. HadjTahar, H. Benali, A. Karni, LG Ungerleider, J. Carrier Contribution of night and day sleep vs. simple passage of time to the consolidation of motor sequence and visuomotor adaptation learning. Experimental Brain Research 2009 May;195(1):15-26.

[26] M. Balas, N. Roitenberg, N, Giladi \& A. Karni. When practice does not make perfect: well-practiced handwriting interferes with the consolidation phase gains in learning a movement sequence. Experimental Brain Research 2007 178(4):499-508.

[27] O. Hikosaka, H. Nakahara, MK. Rand, K. Sakai, K. Nakamura, S. Miyachi, K. Doya: Parallel neural networks for learning sequential procedures. Trends Neurosci 1999, 22:464-471.

[28] DB. Willingham. A neuropsychological theory of motor skill learning. Psychol.Rev. 1998; 105: 558-84.

[29] AS. Keuroghlian, \& EI. Knudsen, Adaptive auditory plasticity in developing and adult animals. Prog. Neurobiol. 82, 109-121 (2007)

[30] DA. Rosenbaum, RA. Carlson, RO. Gilmore. Acquisition of intellectual and perceptual-motor skills. Annu Rev Psychol. 200152:453-70. 